\title{
Chronic cough and wheeze in children: do they all have asthma?
}

\author{
M. Seear, D. Wensley
}

\begin{abstract}
Chronic cough and wheeze in children: do they all have asthma? M. Seear, D. Wensley. CERS Journals Ltd 1997.

ABSTRACT: In the experience of both authors, children referred for investigation of a chronic productive cough often do not fit conventional diagnostic categories. The aim of this study was to answer two questions: 1) do such diagnostic orphans exist? and, 2) if so, can they be classified in a clinically useful manner?

Eighty one previously undiagnosed children referred with a history of more than 3 months productive or rattly cough were studied prospectively. Investigations consisted of a detailed history, physical examination, and an extensive set of clinical investigations. Sixty randomly selected asthmatic children served as demographic controls.

Children fell into three groups: 23 had newly diagnosed conditions, such as cystic fibrosis, or were indistinguishable from asthma; 24 had a history of major medical interventions (cardiac surgery, chemotherapy, tracheo-oesophageal fistula repair); 34 had a history of significant early respiratory tract infections, usually combined with poor social conditions. The latter group differed significantly from asthmatic controls in a wide range of demographic and clinical comparisons. Native American children were overrepresented

The results of this study suggest that early respiratory insults (whether viral or medical) can induce self-perpetuating inflammation, manifesting as a chronic productive cough and intermittent wheeze. The associations between poverty and infant chest infections or early invasive medical treatment with subsequent chronic respiratory disease are clearly definable and probably justify diagnostic terms distinct from asthma, particularly for use in epidemiological studies. Eur Respir J 1997; 10: 342-345.
\end{abstract}

Dept of Respiratory Medicine and Intensive Care, British Columbia's Children's Hospital, Vancouver, British Columbia, Canada

Correspondence: M. Seear

Intensive Care Unit

British Columbia's Children's Hospital 4480 Oak Street

Vancouver

British Columbia

Canada V6H 3V4

Keywords: Asthma chronic bronchitis poverty

Received: April 21996

Accepted after revision October 71996
The classification of children with chronic or recurrent wheezing episodes has always been a contentious issue. In the past, such terms as asthmatoid bronchitis [1], chronic bronchitis [2] and wheezy bronchitis [3] were used to describe poorly-defined conditions with common clinical features of cough and wheeze. The confusion probably contributed to the well-reported misdiagnosis [4] and inappropriate treatment of asthma [5]. These terms were subsequently considered to have little clinical value [6], and were slowly abandoned in favour of "asthma" as a single diagnosis for children with recurrent wheezing.

In the absence of a widely-accepted clinical definition of asthma, controversy persists over the classification of wheezy children. CONNETT [7] divided the current debate into "lumpers", who would include all wheezy children within the umbrella of asthma [8], and "splitters", who believe there are definable subgroups of asthma that deserve separate diagnostic terms $[9,10]$.

In the experience of both authors, there are appreciable numbers of children referred to a paediatric respiratory clinic who do not conform to any conventional diagnostic categories. The hallmark of such patients is a chronic productive or rattly cough, usually accompanied by palpable chest crackles, audible wheezing, and recurrent hospital admissions throughout winter. There is frequently a history either of poor social conditions or previous complex medical interventions.

This simple descriptive study was designed to answer two questions: 1) are there significant numbers of children with chronic productive coughs who do not fit the usual disease categories, particularly asthma? and 2) if such diagnostic orphans exist, can they be classified in a clinically useful manner?

\section{Methods}

\section{Patient selection}

Over a 2 year period (December 1993 to December 1995), all 81 children referred to the respiratory clinic of British Colombia's Children's Hospital, who fulfilled the following criteria, were prospectively studied: 1) productive or rattly cough, with or without wheezing, on most days for three consecutive months or more; and 2) exclusion of children with known causes of productive cough (cystic fibrosis, immunodeficiencies, bronchiectasis and bronchopulmonary dysplasia). 
Similar criteria were used in a study of paediatric bronchitis in Lisbon [11]. The time period was chosen to exclude children with a chronic cough following a respiratory tract infection which, in our experience, usually lasts less than 2 months. Referrals were not solicited. Over the same time period, 60 children who fulfilled our definition of asthma, were randomly selected, based on their hospital numbers, to act as an epidemiological control group.

\section{Investigations}

Patients selected from the two study groups (chronic productive cough and asthmatic controls) underwent a complete history and physical examination, that included standardized, demographic questions. All children in the productive cough group were then investigated fully to determine, as far as possible, a reason for the cough. Each child completed chest radiograph, pulmonary function tests (if old enough), Mantoux test, sweat chloride test, full blood count, and immunoglobulin levels. If clinically indicated, subsequent tests included chest computed tomography (CT)-scan, flexible or rigid bronchoscopy, expanded immune investigations and lung biopsy. No institutional approval was given to perform any tests in asthmatics other than those immediately indicated by the history and physical examination.

\section{Definition of asthma}

Despite an extensive literature, there is no single definition of asthma that satisfies everyone. Prior to referral, all patients had been examined by at least one physician, making it possible to base the definition on a historical set of clinical observations. Asthma was diagnosed when the following criteria were fulfilled: 1) episodic or chronic wheeze, with or without a nonproductive cough, noted on examination by a referring physician; 2) exacerbation of cough and wheeze by upper respiratory tract infections, exercise or airborne allergens; and 3) favourable response to conventional asthma therapy.

\section{Statistical analysis}

Discrete variables are expressed as absolute numbers for each group with percentage occurrence given in parentheses. The variables were compared using Fisher's exact test. Continuous variables are expressed as mean \pm SD and were compared by unpaired t-test. A p-value of less than 0.01 was considered significant for all tests, in order to compensate for the increased chance of a type I error due to the use of multiple comparisons.

\section{Results}

\section{Final diagnoses: chronic productive cough cohort}

Despite extensive investigations and the presence of significant pulmonary disease, surprisingly few of the
Table 1. - Final diagnoses made in 81 children referred with a history of more than 3 months productive cough

\begin{tabular}{lc}
\hline Underlying pathology & $\begin{array}{c}\text { Pts } \\
\mathrm{n}\end{array}$ \\
\hline Paediatric chronic bronchitis* & 34 \\
Indeterminate & 14 \\
Prior complex oncology treatment* & 12 \\
Prior complex cardiac surgery* $^{*}$ & 8 \\
Prior tracheo-oesophageal fistula repair & 4 \\
Idiopathic bronchiectasis & 3 \\
Immunodeficiency & 2 \\
Ciliary dyskinesia & 1 \\
Foreign body & 1 \\
Tuberculosis & 1 \\
Cystic fibrosis & 1 \\
Total & 81 \\
\hline
\end{tabular}

Patients with known pre-existing cystic fibrosis, bronchopulmonary dysplasia or immunodeficiencies were excluded from the study. *: 2 Down's syndrome; +: 1 Down's syndrome.

81 patients could be fitted into a conventional diagnostic category (table 1). They broadly fell into one of three groups.

The first group contained 23 patients with conventional diagnoses. Nine had clearly defined conditions ranging from immunodeficiencies to an unrecognized foreign body. The remaining 14 fulfilled the criteria for asthma except for the presence of a productive, rather than a nonproductive cough, and were classified as "indeterminate".

In the second group of 24 children, the only abnormality discovered was a common past history of significant medical interventions. Two general patterns emerged: 12 children were survivors of complex cancer chemotherapy, and the remainder had a history of complicated postoperative courses following either cardiac surgery ( 8 children) or repair of a tracheo-oesophageal fistula (4 children). The descriptive term "iatrogenic lung disease" (ILD) was used to refer to this group.

The third group of 34 children shared several demographic and clinical features, that clearly distinguished them from the asthmatic control group (table 2). This included a low incidence of eczema and asthmatic family history, and a high incidence of poor social conditions and hospital admission in infancy for pneumonia. This group was considered sufficiently different from the asthmatic controls to justify the use of a separate diagnostic term "paediatric chronic bronchitis" (PCB).

It is interesting to note that children with Down's syndrome were overrepresented in four of the diagnostic subgroups.

\section{Clinical comparison: asthma and $P C B$}

Table 2 shows the results of demographic and clinical comparisons between the 34 children with PCB and the control group of 60 children with asthma.

Compared to the asthmatic controls, a typical child in the PCB group was more likely to be a male from a poor social background; nearly $75 \%$ of the mothers smoked, and the father was absent or unemployed in over $50 \%$ of the cases. Hospital admission for pneumonia in 
Table 2. - Comparative clinical data for 34 cases of paediatric chronic bronchitis and 60 asthmatic controls

\begin{tabular}{lrrrrc}
\hline & \multicolumn{2}{c}{ PCB } & \multicolumn{2}{c}{ Asthma } & p-value \\
\hline Pts n & \multicolumn{2}{c}{34} & \multicolumn{2}{c}{60} & \\
Sex M/F (male \%) & $26 / 8$ & $(76)$ & \multicolumn{2}{c}{$29 / 31(48)$} & $<0.01$ \\
1st symptoms yrs & \multicolumn{2}{c}{$0.5 \pm 0.7$} & \multicolumn{2}{c}{$2.5 \pm 2.4$} & $<0.001$ \\
1st presentation yrs & \multicolumn{2}{c}{$0.8 \pm 0.7$} & \multicolumn{2}{c}{$3.2 \pm 2.4$} & $<0.001$ \\
Hospital administration $<1$ yr & 21 & $(62)$ & 4 & $(7)$ & $<0.001$ \\
Chronic otitis media* n & 14 & $(41)$ & 1 & $(2)$ & $<0.001$ \\
Eczema n & 3 & $(9)$ & 19 & $(32)$ & $<0.01$ \\
Family history of asthma\# n & 5 & $(15)$ & 37 & $(62)$ & $<0.001$ \\
Maternal smoking n & 25 & $(74)$ & 16 & $(27)$ & $<0.001$ \\
Native American Indian & 8 & $(24)$ & 1 & $(2)$ & $<0.001$ \\
Father absent n & 11 & $(32)$ & 4 & $(7)$ & $<0.01$ \\
Father present but unemployed n & 7 & $(21)$ & 0 & $(0)$ & $<0.001$ \\
Hyperinflation on CXR & 15 & $(44)$ & 26 & $(43)$ & NS \\
Bronchial wall thickening on CXR & 11 & $(32)$ & 51 & $(85)$ & $<0.001$ \\
Streaky densities on CXR & 21 & $(62)$ & 11 & $(18)$ & $<0.001$ \\
Patchy densities on CXR & 15 & $(44)$ & 11 & $(18)$ & $<0.01$ \\
\hline
\end{tabular}

Absolute values are presented and percentage in parentheses, or as mean \pm SD. *: defined as history of tympanic perforation or tympanostomy tubes; \#: defined as the diagnosis of asthma in a first degree relative; $\stackrel{+}{+}$ defined by mother's ethnic group; PCB: paediatric chronic bronchitis; M: male; F: female; CXR: chest radiography; Pts: patients.

infancy was 10 times more likely compared to the asthmatic group. Children of Native Indian parentage were significantly overrepresented. Chronic otitis media, persistent nasal discharge and patchy or streaky densities on chest radiography were frequent coexisting findings, that were significantly more common in the PCB group.

Compared to the PCB group, active eczema and a family history of asthma were four times more common amongst the asthmatics. Bronchial wall thickening on chest radiography was also significantly more common in the asthmatic group.

The children included in the ILD group shared only one similarity: a common past history of complex medical treatment. They were, individually, too diverse in social and medical backgrounds to warrant any comparison to the asthmatic controls.

\section{Discussion}

A cohort of 81 children with chronic productive cough studied, in order to determine, as far as possible, a clinical diagnosis. Despite extensive investigations, at least two-thirds of the group (58 children) did not clearly fit any current paediatric diagnostic category. Twenty four of these children were united by a common history of significant invasive medical therapy at an early age (chemotherapy, cardiac surgery, prolonged mechanical ventilation). The remaining 34 children shared many common features, including poor social backgrounds and hospitalization for significant respiratory tract infections at an early age. In our experience, such children consume large amounts of medical attention. For convenience, we refer to the two groups as: iatrogenic lung disease (ILD) and paediatric chronic bronchitis (PCB), respectively.

Distinguishing between two groups of coughing children using only clinical observation is a difficult task. Argument inevitably arises about whether these are separate diseases or simply asthma exacerbated by poverty and previous medical therapies. Although there are no clinical or social features unique to either group, the low incidences of eczema and family history of asthma, combined with the total social and clinical picture of the children that we have labelled PCB, offer some support for our belief that this is a separate disease entity. Children in the ILD group are too diverse for this category to be anything more than a convenient descriptive term.

Given the complex nature of tracheo-oesophageal fistula repair, prolonged chemotherapy and cardiac surgery, it is not surprising that some of the survivors suffer from chronic respiratory disease. As a group, such children are common visitors to referral respiratory clinics and warrant more research than is currently available $[12,13]$. Individual clinical courses are as variable as the past medical histories but, taken as a group, the term "ILD" reflects the unproven but logical assumption that these children are suffering from the chronic effects of early respiratory insults.

The adverse effects of poor living conditions on respiratory health in children have been known for many years [14]. Whether this effect is due to parental smoking [15], air pollution [16], poor access to health care [17] or poor nutrition [18] is difficult to establish. Chronic lung damage resulting from early chest infections with such organisms as respiratory syncytial virus [19] or adenovirus [20] is also well described. Whatever the exact cause, it seems reasonable to assume that the combination of severe respiratory tract infections in early life and poor social conditions produces, in some children, chronic pulmonary inflammation that becomes self-perpetuating, and is manifested by a chronic productive cough with frequent exacerbations throughout childhood [21].

Whilst it is well-recognized that the acute mortality from respiratory tract infections amongst children of developing countries is roughly 30 times higher than it is in Canada [22], the chronic respiratory morbidity amongst survivors is not widely appreciated. In the experience of both authors, PCB is a common condition amongst poor children worldwide. The overrepresentation of Native 
American Indians amongst children with PCB is difficult to explain. Early adenoviral infection has been incriminated as the cause of chronic, postinfectious respiratory disease in Native American Indians [23], ethnic Finns [24] and New Zealand Polynesians [25]. Whether this represents a genetic predisposition amongst some ethnic groups or whether membership of an ethnic group is acting as a marker for other factors is debatable.

Our hypothesis that early respiratory insults (whether medical or infectious) can cause chronic self-perpetuating respiratory inflammation is supported by two recent bronchoscopic studies. HeINo et al. [26] studied seven children referred because of a productive cough lasting more than 3 months, and GAILlARD et al. [27] studied 31 children referred with a loosely defined history of recurrent bronchitis. In both cases, bronchoscopy revealed chronic airway inflammation. Biopsies showed surface epithelial loss with oedema and lymphocytic inflammation in the submucosa. We were only able to justify the use of flexible bronchoscopy in six of the 34 children with PCB. However, our findings of lymphocytes and bacteria on suction samples are very similar to those of an earlier study by Sмітн et al. [10].

Although the criteria used to define PCB and ILD are relatively crude, we believe there is sufficient evidence to separate these children from a cohort of asthmatics. Apart from differences in management and prognosis, our main justification for defining separate terms is to avoid losing valuable clinical data in epidemiological studies. For example, there is good evidence to suggest that early childhood infections, in the setting of poor social conditions (i.e. PCB), predispose a child to the development of chronic bronchitis in adulthood [28, 29]. Large scale prospective studies to determine the origins of this disabling condition will be limited if all coughing and wheezing children are labelled as asthmatics.

\section{References}

1. Kubo S, Funabashi S, Vehara S. Clinical aspects of asthmatic bronchitis and chronic bronchitis in infants and children. J Asthma Res 1978; 15: 99-109.

2. Peat J, Woolcock A, Leeder S. Asthma and bronchitis in Sydney school children. Am J Epidemiol 1980; 111: 721-727.

3. Williams M, McNichol K. Prevalence, natural history and relationship of wheezy bronchitis and asthma in children: an epidemiological study. Br Med J 1969; 4: 321-325.

4. Anderson H, Cooper J, Bailey P. Influence of morbidity, illness label, and social, family and health service factors on drug treatment of childhood asthma. Lancet 1981; ii: $1030-1032$.

5. Speight A, Lee D, Hey E. Underdiagnosis and undertreatment of asthma in childhood. $\mathrm{Br}$ Med J 1983; 286: $1253-1255$.

6. Taussig L, Smith S, Blumenfeld R. Chronic bronchitis in childhood: what is it? Pediatrics 1981; 67: 1-5.

7. Connett G. Acute wheezy bronchitis: lumping and splitting. Arch Dis Child 1991; 66: 751-752.

8. Lee D, Winslow N, Speight A, Hey E. Prevalence and spectrum of asthma in childhood. $\mathrm{Br}$ Med $J$ 1983; 286: $1256-1258$.

9. Wilson N. Wheezy bronchitis revisited. Arch Dis Child 1989; 64: 1194-1199.
10. Smith T, Ireland T, Zaatari G. Characteristics of children with endoscopically proven chronic bronchitis. Am J Dis Child 1985; 139: 1039-1044.

11. Amarla-Marques R, Cochito ME, Cruz M. Prevalence of chronic bronchitis in the children of Lisbon. BronchoPneumologie 1980; 30: 361-366.

12. Chetcuti P, Phelan P, Greenwood R. Lung function abnormalities in repaired oesophageal atresia and tracheo-oesophageal fistula. Thorax 1992; 47: 1010-1014.

13. Shaw N, Tweeddale P, Eden O. Pulmonary function in childhood leukemia survivors. Med Pediatr Oncol 1989; 17: 149-154.

14. Colley J, Douglas J, Reid D. Respiratory disease in young adults: influence of early childhood lower respiratory tract illness, social class, air pollution and smoking. Br Med J 1973; 3: 195-198.

15. Fergusson D, Horwood L, Shannon F. Parental smoking and respiratory illness in infancy. Arch Dis Child 1980; 55: 358-361.

16. Colley J, Reid D. Urban and social origins of childhood bronchitis in England and Wales. Br Med J 1970; 2: 213-217.

17. Schwartz E, Kofie V, Rivo M. Black/white comparisons of deaths preventable by medical intervention: United States and the District of Columbia 1980-1986. Int J Epidemiol 1990; 19: 591-598.

18. Schwartz J, Weiss S. Dietary factors and their relation to respiratory symptoms: the second national health and nutrition examination survey. Am J Epidemiol 1990; 132: $67-76$.

19. Hall C, Hall W, Gala C. Long-term prospective study in children after respiratory syncytial virus infection. $J$ Pediatr 1984; 105: 358-364.

20. Macek V, Sorli J, Kopriva S, Marin J. Persistent adenoviral infection and chronic airway obstruction in children. Am J Respir Crit Care Med 1994; 150: 7-10.

21. Barker D, Osmond C. Childhood respiratory infection and adult chronic bronchitis in England and Wales. $\mathrm{Br}$ Med J 1986; 293: 1271-1275.

22. Chretien J, Holland W, Macklem P, Murray J, Woolcock A. Acute respiratory infections in children. $N$ Engl $J$ Med 1984; 310: 982-984.

23. Herbert F, Wilkinson D, Burchak E. Adenovirus type 3 pneumonia causing lung damage in childhood. Can Med Assoc J 1974; 116: 274-276.

24. Simila S, Linna O, Lanning P. Chronic lung damage caused by adenovirus type 7: a 10 year follow-up study. Chest 1981; 80: 127-131.

25. Lang W, Howden C, Laws J. Bronchopneumonia with serious sequelae in children with evidence of adenovirus type 21 infection. Br Med J 1969; 1: 73-77.

26. Heino M, Juntunen-Backman K, Leijala M, Rapola J, Laitinen L. Bronchial epithelial inflammation in children with chronic cough after early lower respiratory tract illness. Am Rev Respir Dis 1990; 141: 428-432.

27. Gaillard D, Jouet J-B, Egreteau L. Airway epithelial damage and inflammation in children with recurrent bronchitis. Am J Respir Crit Care Med 1994; 150: 810-817.

28. Samet J, Taga I, Speizer F. The relationship between respiratory illness in childhood and chronic airflow obstruction in adulthood. Am Rev Respir Dis 1983; 127: 508-523.

29. Barker D, Osmond C, Law C. The intrauterine and early postnatal origins of cardiovascular disease and chronic bronchitis. J Epidemiol Commun Health 1989; 43: 237-240. 